\title{
Advanced Eyewear with Embedded Hardware for Woman Safety
}

\author{
Narendhiran.S ${ }^{\mathrm{a}}$, Radhakrishnan.P ${ }^{\mathrm{b}}$, and Elangovan. $\mathrm{D}^{\mathrm{b}}$ \\ ${ }^{a} U G$ Scholar, Dept of CSE, Panimalar Engineering College, Chennai, India \\ ${ }^{\mathrm{b}}$ Associate Professor, Dept of CSE, Panimalar Engineering College, Chennai, India
}

\begin{abstract}
Human have always lived for the betterment of themselves but the bitter truth is that the process of modernisation has never focussed on making a safe society for women. The lack of self-reliance in women is one of the shortcomings of modernisation. An Advanced eyewear with an embedded camera will remove the barriers in a woman's life. The combination of wireless communication with IoT sensors increases its scope of use. The People under threat would be intervened with the security officials or alert the emergency contacts saved previously. This paper introduces a security purpose eyewear with the required embedded technology to enhance the life style of a woman. This would bring in quicker and reliable information to the security officials. This would eventually hamper the victimizer from his cruel intentions by creating a fear of being watched.
\end{abstract}

Keywords: self-reliance, wireless communication, navigation, threat detection, sensors.

\section{Introduction}

A sensor is an equipment which can detect changes in its surroundings and transmit the collected data to other electronic devices. Sensors are mostly used as auxiliary devices in a machine. If we look around us there would hardly be any device without an embedded sensor. One would find them in touch-sensitive elevator buttons i.e., the tactile sensor and in the automobiles to detect fast moving objects and perhaps in almost in everyday utility devices. Due to the recent advancements in technology especially in micro machinery, the uses of sensors are now found in new fields. One would still find the traditional sensors such as potentiometers to be still widely used. In most devices, the sensors are found to be the cheap and efficient components. They are easily replaceable when damaged[2][3]. They are manufactured in such a way that they are durable and their efficiency of its output is consistent. They were initially introduced in the $1800 \mathrm{~s}$ for sensing temperature based on the criteria of copper resistance. Since then, the sensors have changed the world. They are used to provide information about the presence or absence of a physical object. They simulate the were

${ }^{1}$ Radhakrishnan P, Assistant Professor, Dept of CSE, Panimalar Engineering College, Chennai, India Emailid : rksiva13@gmail.com 
human senses to detect the presence of a special physical object. For example, Ultrasound sensors respond to reflected sound waves and the information collected is then used to measure distance[2].

\section{A. Auditory sensor}

As the name suggests these sensors detect sound and respond by giving information about the sound level. The operation of these sensors is similar to the processes in the human body that involves our ears and signals being sent to the brain[3]. Measurement of sound is done in decibels $(\mathrm{dB})$. When a person is found to be talking in a normal voice, the sound levels produced from microphone on an average is between $\sim 40$ to 60 $\mathrm{dB}$, whereas when the person is talking loudly, the membrane of the microphone vibrates more rapidly reaching sound levels to about $90 \mathrm{~dB}$.

\section{B. Pulse Sensor and CMOS Sensor}

Companies like Arduino provide the pulse detection sensors which uses the concept of monitoring the heart rate. A heart rate monitor (HRM) could be any device that measures and displays the in real time or record the heart rate for later study. It is largely used to collect heart rate data while performing various types of physical exercises. heart rate. It is mostly used to monitor the heart rate while the person is performing various types of physical exercises. On Comparing ourselves from the previous generations one would find that taking pictures has become cheaper. Present technology includes sensors such as CMOS sensors which detect photons from the surroundings and convert it into electrical signals. CMOS has lesser manufacturing cost than other technologies such as CCDs. If we compare CMOS with other sensors, we would find that almost all of them use similar technologies. The basic principle of detecting the light and converting the detected data into electrical signals and storing them into a storage media. This results in less area for the capturing of photons than a CCD. To acquire photons even from smaller area micro lens is used which can then focus on the photodiodes. Thus, the aforementioned sensors have made possible the detection and transmission of data from the environment efficiently.

\section{Existing System}

In this modern era, sensors[4] have become the requisites of any luxury good. They have conquered almost every aspect of human life. Nowadays, they are even used in automobiles, medicine smart phones, malls and home appliances. They have entered into our lives intervening between us and the computers. To upload pictures of oneself and one's lifestyle has become the recent trend. In the current trend, What's more intriguing is that being incognito is considered as absurd by the present young minds. Thus, people try to live in a virtual world denying the reality. They have trained themselves not to trust anyone lest they be harmed. The darker side is that they are in a verge to become unreliable. So, an advanced eyewear would connect the vulnerable to the previously saved emergency numbers whom one may consider as a family member, friend, acquaintance or the security officials. The feeling of insecurity among the majority of the population in the world had led to companies like apple to introduce 
emergency SOS which alerts the emergency contacts through text messages along with location. However, this has not reduced the significant number of crimes around the world due to the inability of people to use it efficiently. A camera embedded in the spectacles would perhaps capture the faces around a vulnerable woman and a basic voice recorder would explain the situation experienced by her. This security system can also be made available to the men who have a fear of facing any threat in their day today life. The Safety of women's is also great issue due to increasing crime at an alarming rate against women across the globe. A GPS based women's safety system is proposed to resolve which has two security feature [11-18]. The system which is turned on to generate two alert, if in case a woman is harassed or when she is in trouble. The project proposed device it helps to turning on the device, if woman thinks that she feels alone while walking on the road or any remote area or some dark alley. The woman can authenticated the devices can start the system by using fingerprint scan.Once the device started recording every minute and every second photo and video are captured and authorized personnel number though out SMS or to corresponding nearby the police station. In case, if stranger hits the woman on the other hand woman falls down and becomes unconscious, if she is not able to do nothing, the device works automatically starts the dual security features. The device is useful for the women in day to day life and also preventing atrocities against women. There are some proposed systems already existing which talk about the advanced spectacles with embedded hardware such as camera and various applicable sensors [1]. But this proposal has never touched the concept of safeguarding modesty. The architecture of the processing never stops with the spectacles or the smart phone but connects to the appropriate individuals or the official security related groups. This would bring in the fear within the oppressor and hamper him/her in their pursuit of personal whims and gains.

\section{Proposed System}

One may always fear the menace of life, property, chastity, family welfare and so on. The most important one in this project is to press a button for ensuring security. This would activate the camera embedded in the spectacles. Eventually, a wireless communication will be established between the user and the accountable authority i.e., it would alert the emergency contacts which were previously saved or the nearest situated police station or the hospital via a text message and the pictures of the surroundings taken by the camera will be continuously sent to the same. The audio receiver embedded in the spectacles would transmit the currently recorded audio to the needed. The person on the other side of communication may inform the required officials if not informed when a dire need is felt The device suggests you new method of how files are stored in the app by applying the existing encryption method and mobile system. The users are not comfortable about the fact that that their extremely private or confidential files can be accessed for various purposes by the eyewear hardware. However, this can never happen until the smart phone is hacked. These are the reasons to protect women's safety and protection. However, users can only use the eyewear for safety purposes. Eyewear is the project which is mainly used for women's safety. Eyewear is mainly used to find strangers who had involved into insensitive activities against women. In India, especially women are unsafe and in order to avoid these problems we are proposing this system to rescue the women from these threats and also to give vulnerable men to boost their confidence under fearful situations. The 
sensory system starts working when there are unexpected movements, abnormal blood pressure or by the manual initiation of the system by the user. The pulse sensor is meant to work in all situations to log the data of one's personal health. In situations of abnormal readings of the pulse sensor, it would notify the appropriate people or user through smart phone to initiate the working of other sensors. This can also be stated through manual pressing of the specified button. The camera embedded in the spectacles will then start recording the environment the user sees and start sending its input to the smart phone which will finally send it to the saved hardware devices through internet[4]. The auditory sensors are auxiliary sensors embedded into the spectacles which record the audio to provide the proper information of what is happening with the user of the spectacles to the appropriate people. The user and the guardian or security authorities are communicated through a smart phone. Initially the user connects the spectacles with the smart phone manually before starting from the home. The security authority will take care thereafter.

\section{System Architecture}

The working process is initiated by pressing a button when an individual feels a potential threat. Then, the camera starts taking pictures and transmit them frequently to the smart phone which are processed by the sensors. The pulse sensor senses the blood pressure if there is any abnormal increase in it. If any changes are sensed, then the location of the individual is sent through smart phone after accessing the GPS. If there are any emergency contacts which were previously saved, then a text is shown on their screens. If the controls are set to the appropriate security officials then a message is sent to them. In addition to the message, the locations with the pictures are transmitted to the respective individuals or groups. Then the people who receive the information can take the best possible actions to stop or mitigate the crime.[Figure:1]

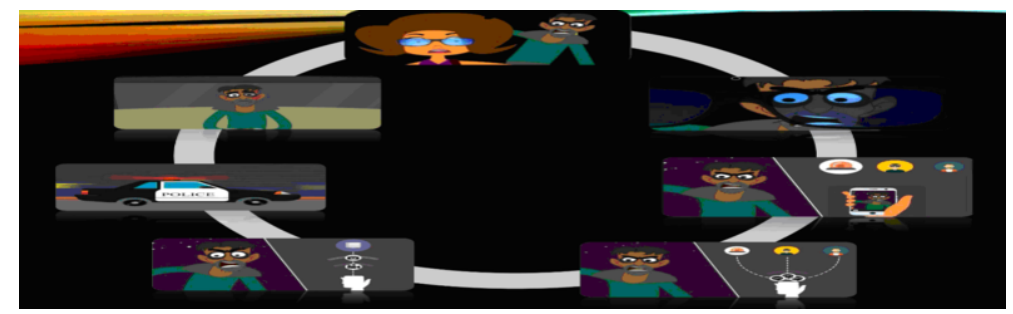

Figure 1. Process of alerting the police

\section{Conclusion And Future Enhancements}

With the advancement of technology, implementing the inventions or discoveries for the maintenance of harmony and peace in the world has been one of the shortcomings of any country. This paper shows a security system for the people who are prone to harassment and danger. It proposes a spectacle which has embedded sensors for sensing abnormal conditions and a camera to capture video in a manner the user perceives the environment about oneself. It would provide the exact location of the victim for faster approach. This device opens the doors towards danger reorganization. 
It breaks the barrier between the victim and the security officials and boosts the speed of acquiring help. This system would bring out the confidence in the people who feel that they are prone to danger. The lives would become simpler with fast communication during inflictions which would finally lead to fear within criminals. Hence, the number of perpetrators of any crime would see a decline within reasonable time. In future this project can be improved further by integrating Aadhaar number into the device. The device can further be made lighter by embedding expensive hardware into the device. This system can also be extended for the general conversations between individuals or groups.

\section{References}

[1] Jianliang Meng, Junwei Zhang,Haoquan . Overview of the Speech Recognition Technology. FourthInternational Conference on Computational and Information Sciences (2019) .

[2] Huang Shan. Voice recognition systems in the telecom prepaid business applications. Information Science(2010).

[3] Alina Munir, Kashif Ehsan S, S. M. Mohsin Raza, Mumajjed Mudassir . Face and Speech Recognition Based Smart Home An offline Security and Automation System (2010) .

[4] Subhas Chandra Mukhopadhyay, Wearable Sensors for Human Activity Monitoring: A Review(2011).

[5] Kiritsis, D., 2011. Closed-loop PLM for intelligent products in the era of the Internet of things. Computer-Aided Design, 43(5), pp.479-501.

[6] Stojkoska, B.L.R. and Trivodaliev, K.V., 2017. A review of Internet of Things for smart home: Challenges and solutions. Journal of Cleaner Production, 140, pp.1454-1464.

[7] Ejaz, W., Naeem, M., Shahid, A., Anpalagan, A. and Jo, M., 2017. Efficient energy management for the internet of things in smart cities. IEEE Communications Magazine, 55(1), pp.84-91.

[8] Varshney, U. and Sneha, S., 2006. Patient monitoring using ad hoc wireless networks: reliability and power management. IEEE Communications Magazine, 44(4), pp.49-55.

[9] Cao, H., Leung, V., Chow, C. and Chan, H., 2009. Enabling technologies for wireless body area networks: A survey and outlook. IEEE Communications Magazine, 47(12), pp.84-93

[10] Cloete, A.H., Brown, J.W.K., Booysen, M.J., Steinke, R. and Magedanz, T., 2016. Smart Grid Application using ETSI M2M: Domestic Electric Water Heaters. SAUPEC 2016 Proceeddings.

[11] Ambeth Kumar.V.D, Dr.S.Malathi, V.D.Ashok Kumar (2015).Performance Improvement Using an Automation System for Segmentation of Multiple Parametric Features Based on Human Footprint. for the Journal of Electrical Engineering \& Technology (JEET), vol. 10, no. 4, pp.1815-1821, 2015. [http://dx.doi.org/10.5370/JEET.2015.10.4.1815]

[12] Ambeth Kumar.V.D , S.Ramya , H.Divakar, G.Kumutha Rajeswari .A Survey on Face Recognition in Video Surveillance",Lecturer Notes on Computational and Mechanism, Vol. 30, pp: 699-708, 2019

[13] Ambeth Kumar.V.D, Precautionary measures for accidents due to mobile phone using IOT. Clinical eHealth, Volume 1, Issue 1, March 2018, Pages 30-35.

[14] K. Nanagasabapathy et.al. Validation system using smartphone luminescence. IEEE International Conference on Intelligent Computing, Instrumentation and Control Technologies (ICICICT), Pages: 235 - 239, 6-7 July 2017, Kannur, India

[15] Ambeth Kumar.V.D et.al; . Cloud enabled media streaming using Amazon Web Services. IEEE International Conference on Smart Technologies and Management for Computing, Communication, Controls, Energy and Materials (ICSTM), Pages: 195-198, 2-4 Aug. 2017, (DOI: 10.1109/icstm.2017.8089150)

[16] Aravindh.B et.al, .A novel graphical authentication system for secure banking systems. IEEE International Conference on Smart Technologies and Management for Computing, Communication, Controls, Energy and Materials (ICSTM), Pages: 177 - 183, 2-4 Aug. 2017, Vel Tech University, Chennai, India

[17] Ambeth Kumar.V.D et.al, “An Efficient Security System for Data base Management from Illegal Access. IEEE International Conference on Wireless Communications, Signal Processing and Networking (WiSPNET), SSN Engineering College, Chennai, India, 23-25 March, 2016

[18] Ambeth Kumar.V.D and Dr.M.Ramakrishan (2011).Web Cam Motion Detection Surveillance System Using Temporal Difference and Optical Flow Detection with Multi Alerts. (CCIS) Journal (Springer) Volume 147, Part 3,pp: 214-221, 2011, April 2011 and the paper was published in the International Journal. [Impact Factor: 0.247].[DOI: 10.1007/978-3-642-20573-6_34] 